\title{
DESCRIPCION DE LA HEMBRA Y DE LAS ESTRUCTURAS GENITALES DE CHILESIA ANGULOI RUIZ (LEPIDOPTERA: ARCTIIDAE)
}

\section{DESCRIPTION OF THE FEMALE AND GENITAL STRUCTURES OF CHILESIA ANGULOI RUIZ (LEPIDOPTERA: ARCTIIDAE)}

\author{
Héctor A. Vargas ${ }^{1} \&$ Luis E. Parra ${ }^{2}$ \\ ${ }^{1}$ Laboratorio de Entomología, Facultad de Agronomía, Universidad de Tarapacá, Casilla 6-D, Arica. E-mail: \\ havargas@uta.cl \\ ${ }^{2}$ Departamento de Zoología, Facultad de Ciencias Naturales y Oceanográficas, Universidad de Concepción, Casilla \\ 160-C, Concepción. E-mail: luparra@udec.cl
}

\section{RESUMEN}

Se describe la morfología externa de la hembra y la genitalia del macho y de la hembra de Ch. anguloi Ruiz. Los caracteres morfológicos de la genitalia del macho permiten distinguir a esta especie de las restantes que componen el género Chilesia Ruiz: Ch. rudis (Butler) y Ch watsoni Ruiz. El carácter braquíptero de la hembra de Ch. anguloi confirma lo descrito previamente para el género. La hembra de Ch. anguloi puede ser claramente diferenciada de las hembras de Ch. rudis y Ch. watsoni, sobre la base de caracteres morfológicos externos y genitales.

Palabras claves: Chilesia watsoni, Genitalia, Braquiptería, Chile.

\begin{abstract}
Female external morphology and male and female genitalia of $C h$. anguloi Ruiz are described. This species can be clearly differentiated from the other species of Chilesia Ruiz: Ch. rudis (Butler) y Ch. watsoni Ruiz, through morphological characters of the male genitalia. Female brachyptery of Ch. anguloi confirms that formerly described for the genus. However, the female of $\mathrm{Ch}$. anguloi can be easily distinguished from females of Ch. rudis y Ch. watsoni, on the base of the external morphology and genital characters.
\end{abstract}

KeYwords: Chilesia watsoni, Genitalia, Brachyptery, Chile.

\section{INTRODUCCION}

Arctiidae es la segunda familia más diversa de Noctuoidea (Nielsen \& Common 1991). En Chile se encuentra representada por al menos 14 especies, repartidas en 10 géneros (Ruiz 1989). El género
Chilesia Ruiz es endémico del territorio chileno, y tres son las especies que lo conforman: Ch. anguloi Ruiz, Ch. rudis (Butler) y Ch. watsoni Ruiz (Ruiz 1989).

Ch. rudis y Ch. watsoni son dos especies bien conocidas en lo que se refiere a la morfología 
externa de sus imagos, y a la morfología de las estructuras genitales del macho y de la hembra. Ambas especies presentan machos con alas bien desarrolladas y hembras braquípteras (Ruiz 1989). Adicionalmente, para ambas especies se conoce la morfología y algunos aspectos de la biología de los estados inmaduros (Angulo \& Ruiz 1975; Ruiz 1989).

Ch. anguloi fue descrita exclusivamente sobre la base de caracteres morfológicos externos del macho (Fig. 1b). De esta manera, la hembra permaneció desconocida hasta ahora, al igual que la morfología genital de ambos sexos.

En el presente trabajo se describe e ilustra por primera vez la morfología externa de la hembra y las estructuras genitales del macho y de la hembra de Ch. anguloi.
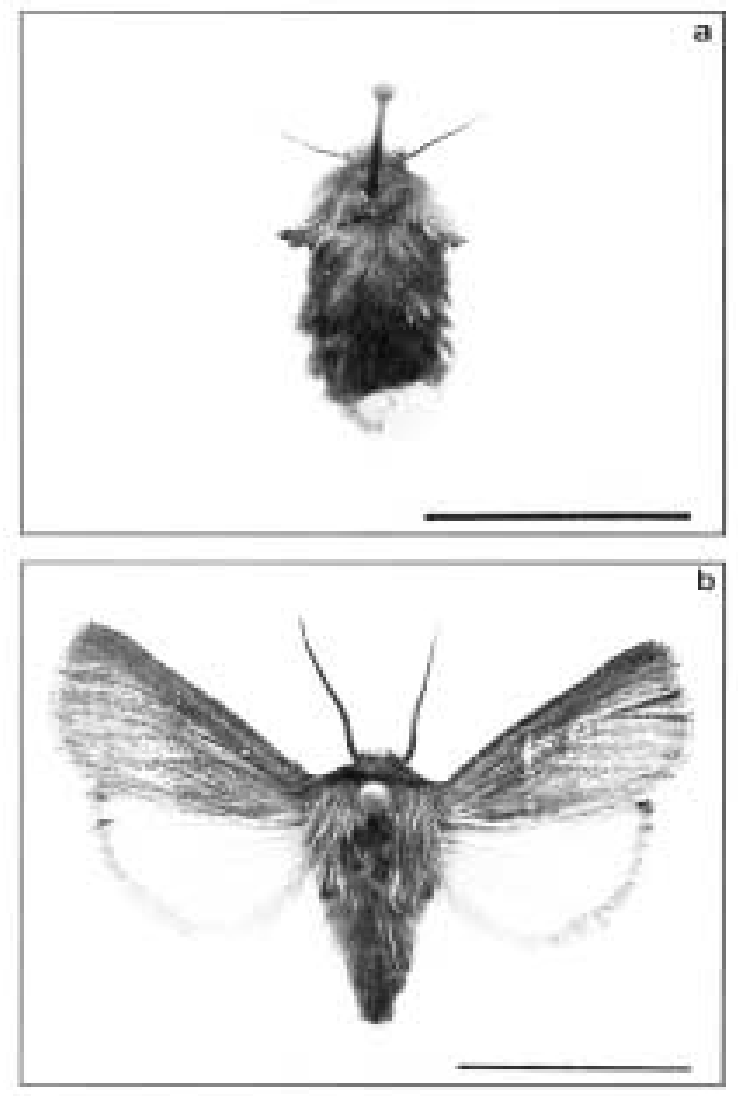

Figura 1. Imagos de Chilesia anguloi Ruiz. a) dorsal; y b) ơ vista dorsal. Escala $1 \mathrm{~cm}$.

Figure 1. Adults of Chilesia anguloi Ruiz. a) Female in dorsal view; and b) male in dorsal view. Scale $1 \mathrm{~cm}$.

\section{MATERIALES Y METODOS}

El material examinado corresponde a doce ejemplares depositados en la Colección Entomológica de la Facultad de Agronomía de la Universidad de Tarapacá (CICA). Los procedimientos utilizados para el estudio morfológico corresponden a aquéllos tradicionalmente empleados en taxonomía de Lepidoptera (Bolte, 1990). Para la nomenclatura de las armaduras genitales se siguió a Klots (1970).

\section{RESULTADOS}

\section{DESCRIPCIONES}

Genitalia del macho (Fig. 2a-d). Uncus subtriangular, ligeramente curvo y de ápice romo (Figs. 2a y 2d). Tegumen angosto. Valvas pequeñas (Fig. 2c), subtriangulares; margen dorsal y anterior sinuosos; margen ventral ligeramente invaginado; ápice romo. Saccus angosto y semicircular (Fig. 2a). Yuxta esclerosada, subtrapezoidal; margen ventral redondeado, márgenes laterales sinuosos, margen dorsal invaginado (Fig. 2a). Aedeagus subcilíndrico y ligeramente curvo en la base (Fig. 2b); longitud cerca de dos veces la de las valvas; vesica carece de cornuti, pero está provista de pequeñas espínulas de forma irregular.

Morfología externa de la hembra (Fig. 1a). Braquíptera, cuerpo cubierto de escamas pardas. Antenas ciliadas. Proboscis ausente. Palpos labiales cortos, ocultos por las escamas del penacho frontal. Alas reducidas a muñones. Abundantes escamas blancas piliformes, ligeramente onduladas y muy finas dispuestas en el abdomen en posición subterminal.

Genitalia de la hembra (Fig. 2e). Apófisis anteriores y posteriores ligeramente curvas, bien esclerosadas y similares en longitud. Lamella antevaginalis indiferenciada; lamella postvaginalis conspicua, con el margen posterior lobulado. Antrum conspicuo; con un débil esclerosamiento en la pared dorsal. Ductus bursae cilíndrico y sinuoso; con un apéndice membranoso y dilatado en el extremo distal, que nace sobre la mitad de la longitud del ductus. Corpus bursae piriforme, de longitud superior a la del ductus bursae; carece de signum. Ductus seminalis nace de la base del corpus bursae. 

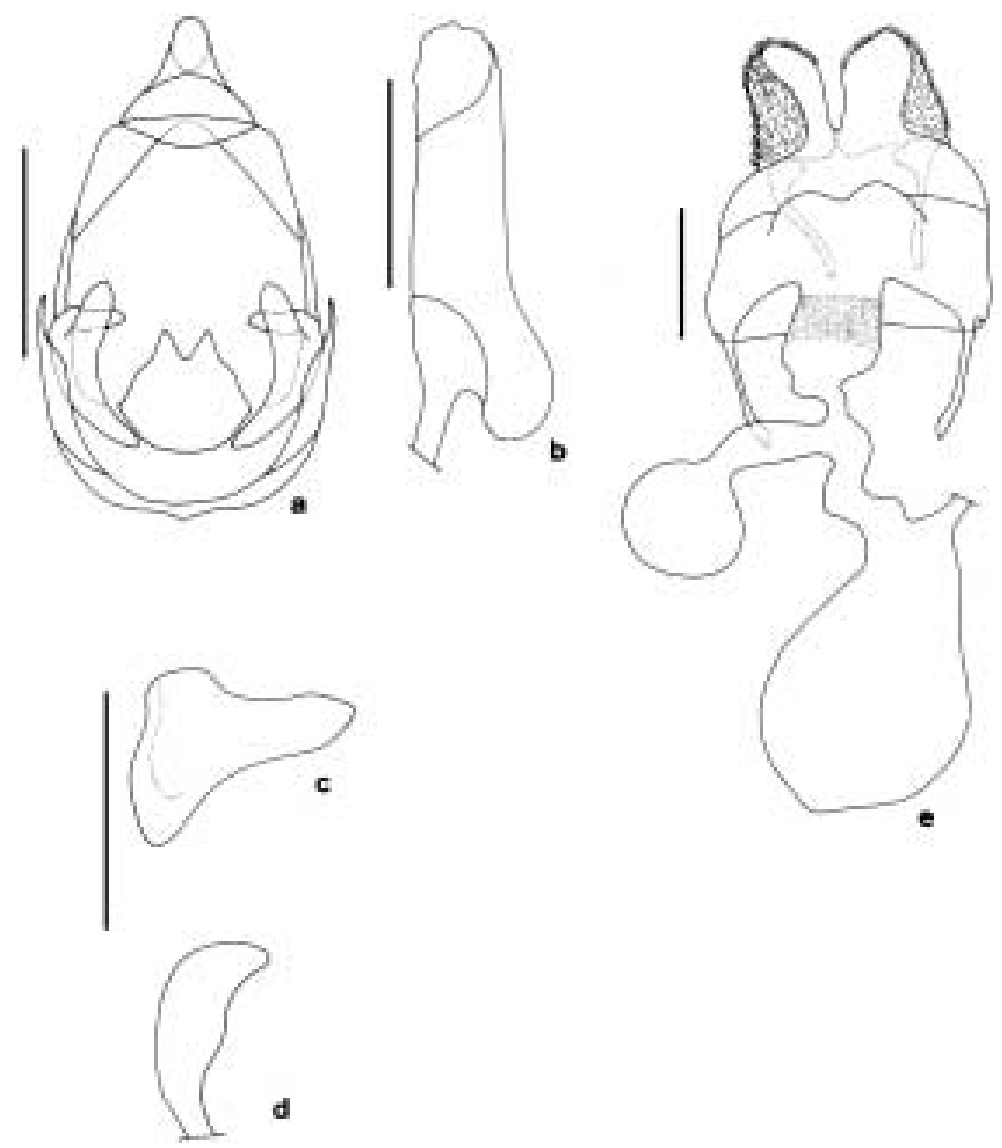

Figura 2. Armaduras genitales de Chilesia anguloi Ruiz. a) Genitalia del macho en vista ventral; b) aedeagus en vista lateral; c) valva en vista lateral; d) uncus en vista lateral; y e) genitalia de la hembra. Escala $1 \mathrm{~mm}$.

Figure 2. Genitalia of Chilesia anguloi Ruiz. a) Male genitalia in ventral view; b) aedeagus in lateral view; c) valva in lateral view; d) uncus in lateral view; and e) female genitalia in dorsal view. Scale $1 \mathrm{~mm}$.

\section{MATERIAL EXAMINADO}

$40^{\star} 0^{\star} ; 8$ ㅇ 우. PARINACOTA. Belén. $20^{\star}:$ ob. lab. 2603-98, en alfalfa. A. Morales coll.; 5 \% : ob. lab. 2603-98, en alfalfa. A. Morales coll. Lupica. $10^{\top} \mathrm{ob}$. lab. 17-04-97, en malezas, H. Vargas C. coll.; 1 o $0^{*}$ ob. lab. 17-04-97, en malezas, R. Mendoza coll.; 1 \% : ob. lab. 17-04-97, en malezas, R. Mendoza coll.; 2 i ob. lab. 13-04-97, en malezas, R. Mendoza coll. (CICA).

\section{Discusión}

Los caracteres morfológicos de la genitalia del macho de Ch. anguloi presentan una notable similitud con los de Ch. rudis y Ch. watsoni. Sin embargo, la invaginación en el margen dorsal de la yuxta de $C h$. anguloi permite separarla claramente de la otras dos especies.

El carácter braquíptero de la hembra de $C h$. anguloi concuerda con lo previamente descrito para las hembras de las restantes especies de este género. Sin embargo, el grupo abdominal subterminal de escamas blancas de la hembra de Ch. anguloi, que contrasta notablemente con las escamas pardas del resto del cuerpo, permite diferenciarla de las hembras de Ch. rudis y Ch. watsoni.

En relación con la genitalia de la hembra de Ch. anguloi, la presencia del apéndice membranoso asociado al ductus bursae es un buen carácter para distinguirla de las otras dos especies de Chilesia. 
Gayana 67(1), 2003

\section{AGRADECIMIENTOS}

Los autores agradecen al entomólogo $\mathrm{Sr}$. Héctor E. Vargas Carreño, por la colaboración prestada en la confección del abstract; y a la Srta. Yubitza J. Cortés Jorquera, por la ayuda brindada en la digitación del texto e impresión de las fotografías. Al Proyecto 200.113.056-1.0 de la Dirección de Investigación de la Universidad de Concepción y al proyecto DIPOG 9704-03, Dirección de Investigación, Universidad de Tarapaca.

\section{BIBLIOGRAFIA}

Angulo, A.O. \& V.H. RuIz. 1975. Maenas rudis (Butler); cuncuna colorada de prados y jardines; biolo- gía y estados inmaduros (Lepidoptera: Arctiidae). Bol. Soc. Biol. Concepción. Chile. 49: 139-147.

Bolte, K.B. 1990. Guide to the Geometridae of Canada (Lepidoptera). VI. Subfamily Larentiinae. 1. Revision of the genus Eupithecia. Memoirs of The Entomological Society of Canada. N 151. $253 \mathrm{pp}$.

KLots, A.B. 1970. Lepidoptera. En: Tuxen SL (ed.). Taxonomist's Glossary of Genitalia in Insects ( $2^{\text {nd }}$ ed.): $115-130$. Munksgaard, Copenhagen.

Nielsen, E.B. \& I.F.B. Common. 1991. Lepidoptera (moths and butterflies). Chapter 41. In: Nauman I.D. (Ed). The Insects of Australia ( $\left.2^{\text {nd }} e d\right) p p$ : 817-915. Melbourne University Press, Carlton, Victoria \& University College of London Press, London.

RuIz, V.H. 1989. Revisión sistemática de la familia Arctiidae en Chile (Lepidoptera). Gayana (Zoología) 53(4): 117-181.

Fecha de recepción: 16/11/02

Fecha de aceptación: 09/05/03 